\title{
Phosphorylation and stabilization of c-Myc by NEMO renders cells resistant to ionizing radiation through up-regulation of $\boldsymbol{\gamma}$-GCS
}

\author{
BU-YEON KIM, SEO-YOUNG KWAK, JI-SOOK YANG and YOUNG-HOON HAN \\ Division of Radiation Cancer Research, Korea Institute of Radiological and \\ Medical Sciences, Seoul 139-706, Republic of Korea
}

Received June 28, 2011; Accepted July 26, 2011

DOI: $10.3892 /$ or.2011.1432

\begin{abstract}
The transcription factor c-Myc has been previously shown to be phosphorylated and stabilized by NEMO through direct interaction in the nucleus. Here, we show that NEMO induces up-regulation of the c-Myc target protein, $\gamma$-glutamyl-cysteine synthetase $(\gamma$-GCS $)$, leading to an increase of intracellular glutathione (GSH) levels and simultaneous enhancement of redox-controlling capacity. NEMO enhanced c-Myc recruitment to $\gamma$-GCS promoters and c-Myc was essential for NEMO-mediated $\gamma$-GCS up-regulation. The phosphorylation and stabilization of c-Myc by NEMO rendered cells more resistant to ionizing radiation (IR). Thus, the interaction between NEMO and c-Myc may be targeted for the development of strategies to overcome the resistance to radiotherapy.
\end{abstract}

\section{Introduction}

The cellular oncoprotein c-Myc, a basic helix-loop-helix/ leucine zipper transcription factor, plays a critical role in a variety of cellular processes including cell cycle progression, proliferation, differentiation and apoptosis. c-Myc is a protein with an approximate half-life of $\sim 30 \mathrm{~min}$ (1), therefore the regulation of protein degradation mediated by ubiquitinationproteasome pathway is one of the most important mechanisms for the regulation of c-Myc activity (2). For example, c-Myc stabilization by phosphorylation-associated inhibition of ubiquitination is involved in tumorigenesis process induced by oncogenic Ras activation $(3,4)$.

c-Myc is known to have two-faced functions. Oncogenic property of c-Myc is emphasized by the general up-regulation of c-Myc expression in most of human cancers during tumor

Correspondence to: Dr Young-Hoon Han, Division of Radiation Cancer Research, Korea Institute of Radiological and Medical Sciences, Nowon-gil 75, Nowon-gu, Seoul 139-706, Republic of Korea

E-mail: yhhan@kirams.re.kr

Key words: c-Myc, NEMO, $\gamma$-glutamyl-cysteine synthetase, ionizing radiation progression (5). Accordingly, many reports have demonstrated that c-Myc prevents apoptosis in response to a variety of stimuli in a various cellular context. Down-regulation of c-Myc increases susceptibility of M14 melanoma cells to cisplatin through reactive oxygen species (ROS)-mediated apoptosis and glutathione (GSH) depletion is a key factor in this apoptosis induction (6,7). c-Myc has also been reported to prevent paclitaxel-induced apoptosis by enhancing paclitaxelinduced endoreduplication (8). Sensitization of non-Hodgkin's lymphoma cells to ionizing radiation by rituximab is achieved through the inhibition of c-Myc expression (9). Another study has suggested that c-Myc down-regulation sensitizes melanoma cells to radiotherapy in a p53-independent fashion by inhibiting MLH1 and MSH2 mismatch repair proteins (10). On the other hand, there have also been many reports evidencing proapoptotic function of c-Myc. c-Myc augments gamma irradiation-induced apoptosis by suppressing Bcl-XL independently of p53 status (11). c-Myc also promotes apoptosis by triggering a proapoptotic mitochondrial destabilizing activity that cooperates with proapoptotic members of the Bcl-2 family, especially Bax (12).

In our recent report, we elucidated a new function of $\mathrm{NF}-\kappa \mathrm{B}$ essential modulator (NEMO)/IKK $\gamma$ as a c-Myc protein stabilizer independent of IKK activation (13). NEMO induces phosphorylation at Thr58/Ser62 and concomitant stabilization of c-Myc protein through direct interaction in the nucleus. This modification and stabilization of c-Myc by NEMO enhances the expression of several known c-Myc targets such as cyclin D1, cyclin E and a heavy chain of $\gamma$-glutamylcysteine synthetase $\left(\gamma-\mathrm{GCS}_{\mathrm{H}}\right)$. Up-regulation of $\gamma-\mathrm{GCS}_{\mathrm{H}}$, a catalytic subunit of an important player of cellular redox control system, suggests the possibility that NEMO influences cellular response to stimuli involving oxidative stress such as ionizing radiation (IR) by regulating the expression of c-Myc target proteins. In this study, we investigated the effect of NEMO-mediated c-Myc phosphorylation/stabilization on the cellular response to IR, and report that NEMO overexpression renders cells more resistant to IR by enhancing the expression of redox-controlling c-Myc target, $\gamma$-GCS. Our results suggest that the interaction between NEMO and c-Myc may serve as a good target for the development of strategy to overcome the resistance to radiotherapy. 

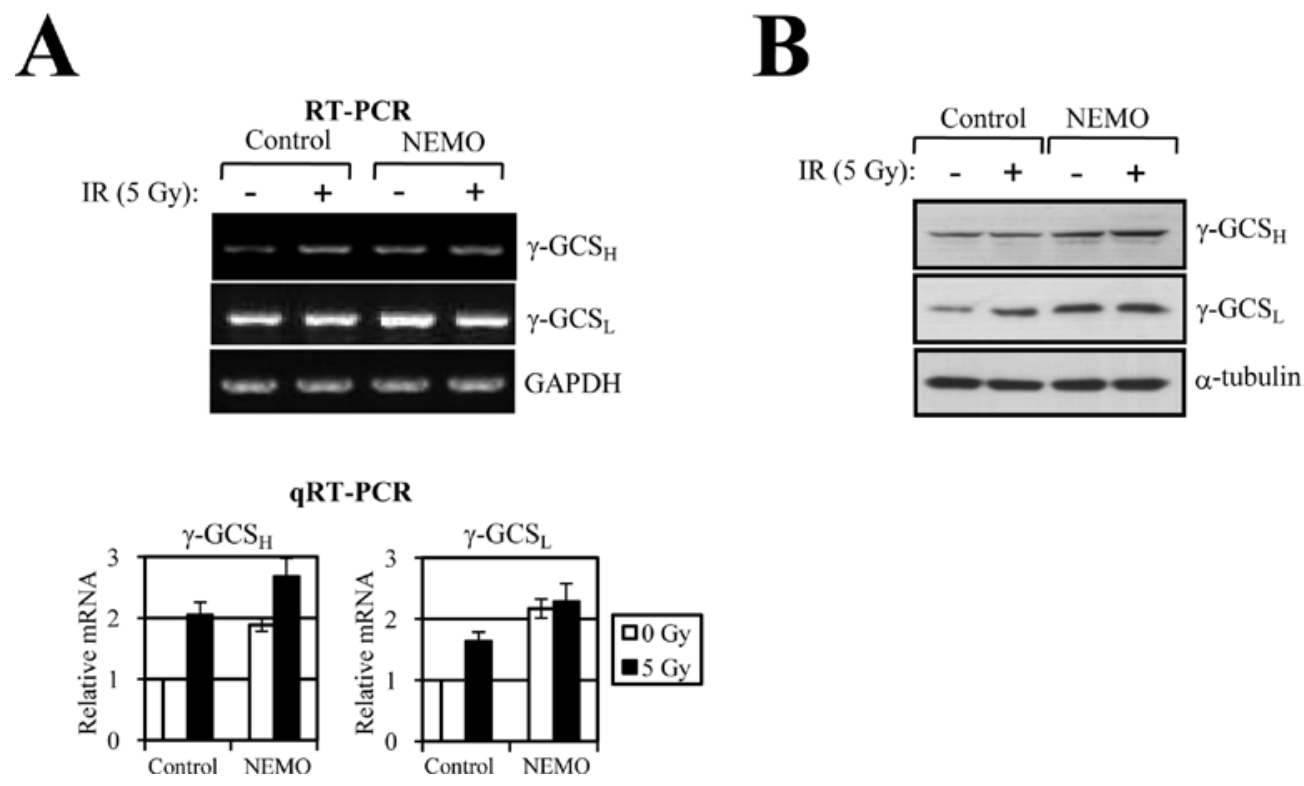

Figure 1. NEMO overexpression enhances the expression of c-Myc targets, $\gamma-\mathrm{GCS}_{\mathrm{H}}$ and $\gamma-\mathrm{GCS}_{\mathrm{L}}$. (A) Overexpression of NEMO enhances mRNA expression of $\gamma-\mathrm{GCS}_{\mathrm{H}}$ and $\gamma-\mathrm{GCS}_{\mathrm{L}}$. HEK293 cells transfected with the control or HA-NEMO expression vector were irradiated at 5 Gy or not. Total RNA was prepared $4 \mathrm{~h}$ after irradiation and mRNA expression for $\gamma-\mathrm{GCS}_{\mathrm{H}}$ and $\gamma-\mathrm{GCS}_{\mathrm{L}}$ was determined by conventional RT-PCR (upper panel) and qRT-PCR (lower panel). GAPDH and $\beta$-actin were used as normalizers for conventional RT-PCR and qRT-PCR, respectively. (B) Enhanced expression of $\gamma$-GCS H $_{\mathrm{H}}$ and $\gamma$-GCS by NEMO was $_{\mathrm{L}}$ confirmed at the protein level. Cell lysates were prepared from the same set of cells used for mRNA determination shown in (A) and analyzed by Western blotting. $\alpha$-tubulin was used for loading control.

\section{Materials and methods}

Cell culture and plasmid constructs. HEK293 was grown in DME medium supplemented with $10 \%$ fetal bovine serum (FBS). Lung cancer cell line H460 was maintained in RPMI-1640 medium supplemented with 10\% FBS. The expression vector for HA-NEMO was described previously (13), and the H460 cell line for stable expression of HA-NEMO was established by retroviral transduction of MFG vector harboring NEMO open reading frame followed by selection in the growth medium containing puromycin at $0.2 \mathrm{mg} / \mathrm{ml}$.

Immunoblotting. Cell lysates were boiled in sodium dodecyl sulfate (SDS) sample buffer, resolved by SDS-polyacrylamide gel electrophoresis ( 8 or $12.5 \%$ polyacrylamide) and transferred to nitrocellulose. After transfer, the membranes were blocked in 5\% milk in TBST (10 mM Tris-HCl, $\mathrm{pH} 8.0$, $150 \mathrm{mM} \mathrm{NaCl}$ and $0.05 \%$ Tween-20) for $30 \mathrm{~min}$ and incubated with primary antibody in 5\% milk in TBST for $2 \mathrm{~h}$ at room temperature. The membranes were washed three times with TBST and incubated for $1 \mathrm{~h}$ at room temperature in TBST containing horseradish peroxidase-linked anti-IgG. After 3 washes in TBST, immunoreactive products were detected by chemiluminescence with an enhanced chemiluminescence system (ECL, Amersham). Antibodies specific for c-Myc (N-262 or 9E10), NEMO (FL-419), $\gamma-\mathrm{GCS}_{\mathrm{H}}(\mathrm{H}-300), \gamma-\mathrm{GCS}_{\mathrm{L}}$ (FL-274), were purchased from Santa Cruz Biotechnology.

$R T-P C R$ and $q R T-P C R$. Total RNAs were extracted using TRIzol reagent (Invitrogen) according to the instructions provided by the manufacturer. Two micrograms of total RNAs were transcribed into single-stranded cDNA using Superscript II (Invitrogen) and serial dilutions of synthesized cDNA were used for following PCR amplification. The primers for $\gamma-\mathrm{GCS}_{\mathrm{H}}$ mRNA were 5'-ATTGTTATGGCTTTGAGTGC-3' (forward) and 5'-CTGTGCTACCTTCATGTTC-3' (reverse). The primer for $\gamma$-GCL $\mathrm{G}_{\mathrm{L}}$ mRNA were 5'-GGAGCATTTACAGCCTTAC-3' (forward) and 5'-CATGAGAATGGATTGATATGG-3' (reverse). GAPDH specific primers used for internal control were 5'-CCATCACCATCTTCCAGGAG-3' (forward) and 5'-CCTGCTTCACCACCTTCTTG-3' (reverse). Quantitative real-time PCR (qRT-PCR) was performed using SYBR Premix Ex Taq II (Takara) and the iCycler real-time PCR detection System (Bio-Rad). The primers for qRT-PCR were as follows: 5'-GAGAACATGAAGGTAGCAC-3' (forward) and 5'-CTTGCTTGTAGTCAGGATG-3' (reverse) for $\gamma-\mathrm{GCS}_{\mathrm{H}} ; 5^{\prime}$-CCTCCTATTGAAGATGGAG-3' (forward) and 5'-TGACCGAATACCGCAGTAG-3' (reverse) for $\gamma-\mathrm{GCL}_{\mathrm{L}}$.

siRNA transfection. Stealth siRNA duplex oligonucleotides targeting c-Myc (si-Myc; 5'-UUCUUGCUCCUCAGAGUC GCUG-3') and scrambled non-targeting negative control siRNA (sc-RNA) were purchased from Invitrogen. Cells were transfected with siRNA oligonucletides at a final concentration of $20 \mathrm{nM}$ using the Oligofectamine Transfection Reagent (Invitrogen) according to the manufacturer's protocol.

Chromatin immunoprecipitation assays. Chromatin immunoprecipitation (ChIP) assay was performed by using a Chromatin Immunoprecipitation Assay kit (Upstate) according to the manufacturer's instruction. Briefly, formaldehyde-crosslinked chromatin fragments were immunoprecipitated with rabbit normal IgG or anti-c-Myc (N-262) antibodies and the specifically precipitated DNA fragments were detected by PCR amplification using the specific primer sets described previously (14). The primers for 
$\gamma-\mathrm{GCS}_{\mathrm{H}}$ E box were 5'-GTGTCCGTGCCATCCAGAGAA CAT-3' (forward) and 5'-GGGACGAGTTCTTCCCATATC TGC-3' (reverse). The primers for $\gamma-\mathrm{GCS}_{\mathrm{L}} \mathrm{E}$ box were 5'-GGG TGCAGCAAACCAACAT-3' (forward) and 5'-GCAAGA ATACTATAATGAAACCCG-3' (reverse). To verify that an equivalent amount of chromatin was used in the immunoprecipitation, PCR reactions were also performed using pre-immunoprecipitation samples representing $0.01 \%$ of the total chromatin (input).

Determination of intracellular GSH and ROS level. Intracellular GSH content was determined using Glutathione Assay Kit (Cayman Chemical) according to the manufacturer's instruction. Intracellular ROS production was measured by staining cells with 2'-7'-dichlorofluorescin diacetate (DCFDA; Molecular Probe). Cells were treated with DCFDA at $1 \mu \mathrm{M}$ in growth medium $20 \mathrm{~h}$ after irradiation and incubated at $37^{\circ} \mathrm{C}$ for $30 \mathrm{~min}$. The changes in fluorescence intensity were monitored by flow cytometry using a FACS Calibur apparatus (BD Biosciences).

Growth inhibition assay. Cells were seeded in 96-well plates and allowed to attach for $24 \mathrm{~h}$, and then irradiated and allowed to grow for $72 \mathrm{~h}$. The number of viable cells was determined using the 3-(4,5-dimethylthiazol-2-yl)5-(3-carboxymethoxyphenyl)-2-(4-sulfophenyl)-2H-tetrazollium inner salt (MTS) reduction assay (Cell Titer 96 AQueous Non-radioactive Cell Proliferation Assay, Promega, Madison, WI) according to the manufacturer's protocol. Three replicates were done for each treatment.

Colony-formation assay. Clonogenic cell survival was evaluated by colony-formation assay. Two hundred cells were seeded on $60-\mathrm{mm}$ dish a day before irradiation. Cells were then irradiated at the indicated doses and allowed to grow to form colonies. After 2 weeks of incubation, cells were fixed with $3.7 \%$ formaldehyde and stained with crystal violet. Colonies $>0.6 \mathrm{~mm}$ in diameter were counted. Three replicates were done for each treatment.

Cell death analysis. Apoptotic cell death was determined by Annexin V-FITC/propidium iodide double staining using Annexin V-FITC Apoptosis Detection Kit I (BD Biosciences) according to the manufacturer's recommendation. The cells were then analyzed using a FACScan flow cytometer (BD Biosciences).

Ionizing radiation of cells. Cells were plated in $3.5-\mathrm{cm}$ dishes and incubated at $37^{\circ} \mathrm{C}$ under humidified $5 \% \mathrm{CO}_{2}-95 \%$ air in culture medium until cells reached $70-80 \%$ confluent. Cells were then exposed to $\gamma$-rays with ${ }^{137} \mathrm{Cs} \gamma$-ray source (Atomic Energy of Canada, Ltd., Canada) with a dose rate of $3.81 \mathrm{~Gy} / \mathrm{min}$.

\section{Results}

NEMO overexpression enhances the expression of $\gamma$-GCS and $G S H$ level, and modulates the redox response to $I R$. We have previously shown that NEMO-induced phosphorylation and concomitant stabilization of c-Myc leads to an increase in the
A

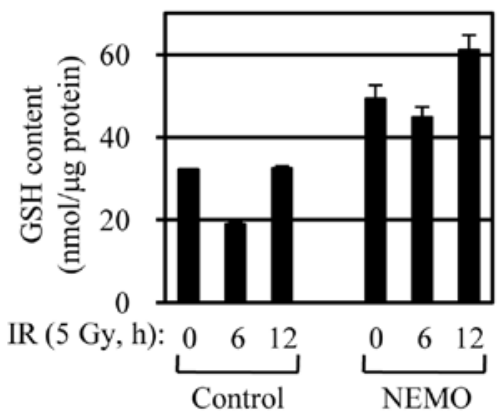

B
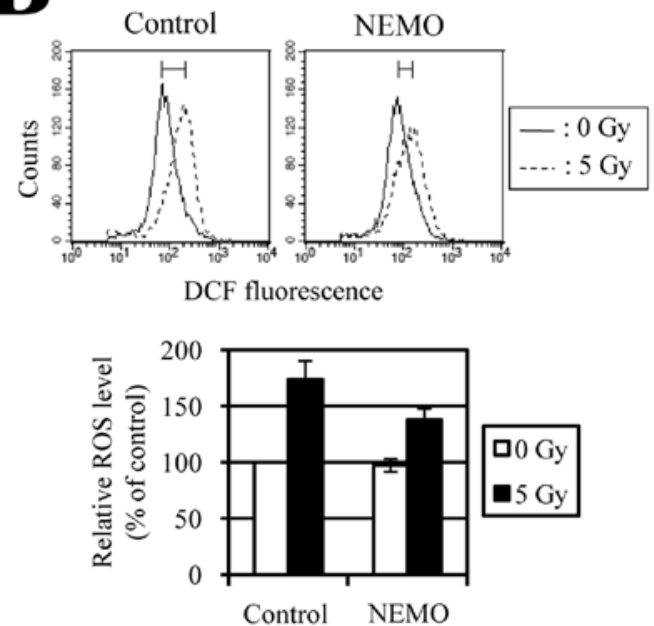

Figure 2. NEMO overexpression induces the increase of intracellular GSH level and alleviates IR-induced increase of intracellular ROS level. (A) Overexpression of NEMO results in the increase of intracellular GSH level. HEK293 cells transfected with the control or HA-NEMO expression vector were irradiated at 5 Gy or not, and GSH contents of the cell lysates prepared 6 and $12 \mathrm{~h}$ after irradiation were determined as described in Materials and methods. (B) Overexpression of NEMO alleviates IR-induced increase of intracellular ROS level. HEK293 cells transfected with the control or HA-NEMO expression vector were irradiated at $5 \mathrm{~Gy}$ or not and stained with DCFDA $20 \mathrm{~h}$ after irradiation. The changes in fluorescence intensity were monitored by flow cytometry.

transcriptional activity of c-Myc and accordingly the expression of many c-Myc target proteins including a heavy subunit of $\gamma$-glutamyl-cysteine synthetase $\left(\gamma-\mathrm{GCS}_{\mathrm{H}}\right)(13)$. In addition to the role in the regulation of cell cycle, differentiation and apoptosis, c-Myc is also known to play a role in the cellular response to oxidative stress by regulating the expression of $\gamma$-GCS, a key enzyme for the first rate-limiting step of glutathione (GSH) biosynthesis $(7,14)$. Since the generation of reactive oxygen species (ROS) is one of the main mechanisms by which IR damages cells, it was highly plausible that NEMO-mediated c-Myc stabilization can modulate the cellular response to IR by regulating the expression of $\gamma$-GCS. Thus, we examined the expression of $\gamma$-GCS in an effort to determine the role of NEMO-mediated c-Myc stabilization in the cellular response to IR. Firstly, we determined the expression of $\gamma-\mathrm{GCS}_{\mathrm{H}}$ and $\gamma-\mathrm{GCS}_{\mathrm{L}}$, constituent subunits of $\gamma$-GCS, by conventional and quantitative RT-PCR. Both basal and IR-induced levels of $\gamma-\mathrm{GCS}_{\mathrm{H}}$ and $\gamma-\mathrm{GCS}_{\mathrm{L}}$ mRNA were increased when NEMO was 
A

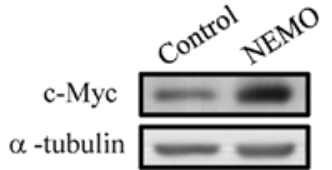

B

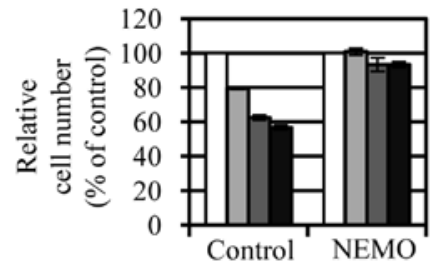

C
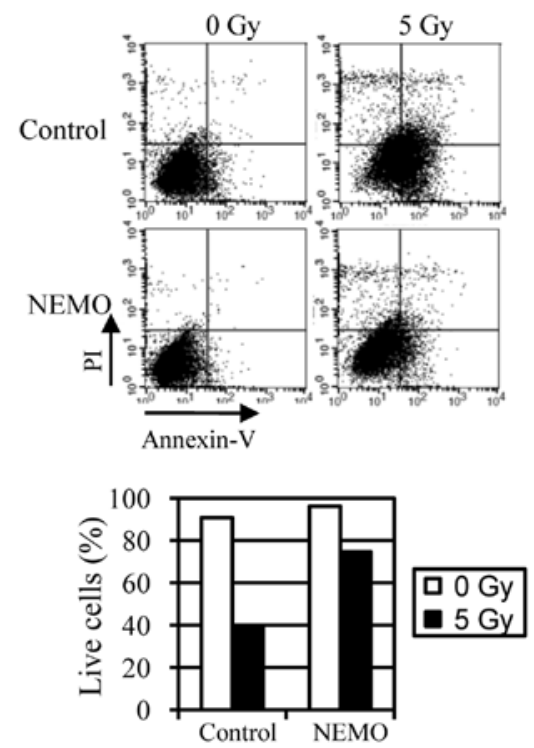
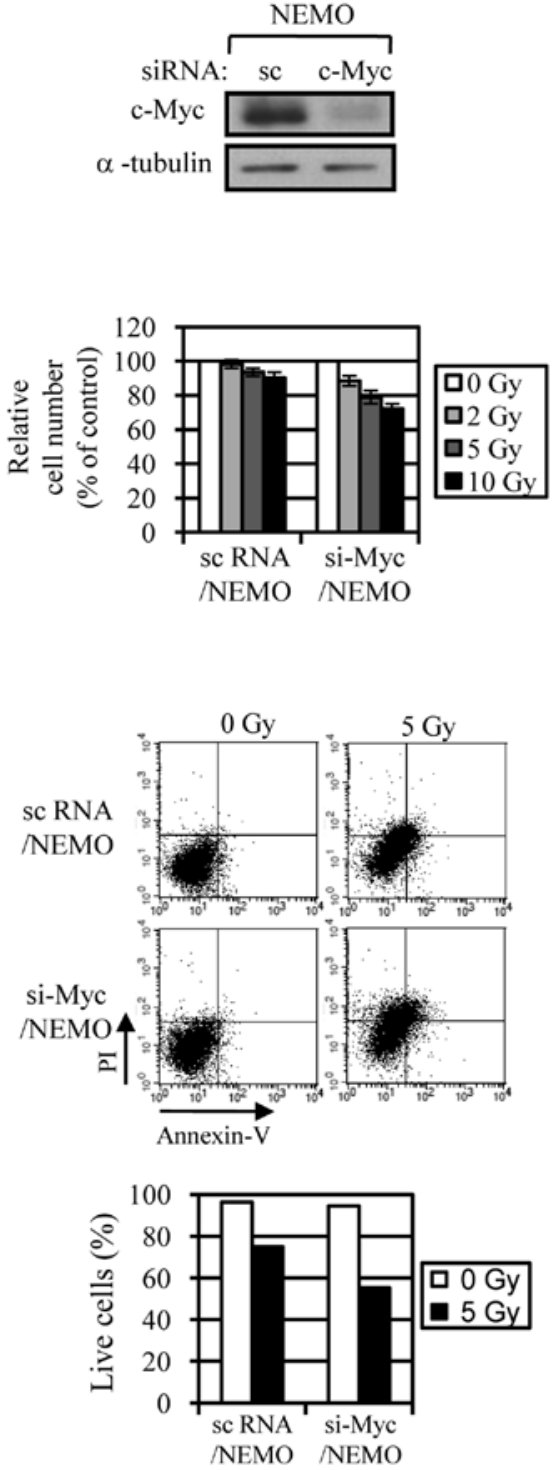

Figure 3. NEMO renders cells resistant to IR through c-Myc up-regulation. (A) Confirmation of c-Myc up-regulation by NEMO transfection and downregulation of c-Myc by specific c-Myc siRNA transfection. HEK293 cells were transfected with the control or HA-NEMO expression vector (left panel). The same cells were transfected with scrambled control or c-Myc specific siRNA together with HA-NEMO expression vector (right panel). (B) Growth inhibition by IR is blocked by NEMO transfection and c-Myc down-regulation by siRNA transfection reverses the effect of NEMO. HEK293 cells transfected as indicated were irradiated at the indicated doses and the relative viable cell numbers were determined $72 \mathrm{~h}$ after irradiation by MTS assay. (C) NEMO transfection protects cells from IR-induced cell death and the protective effect of NEMO is reversed by c-Myc down-regulation. HEK293 cells were transfected and treated as indicated and cell death was determined after $48 \mathrm{~h}$ by Annexin-V/PI staining followed by FACS analysis. The fraction of live cells (lower left section) was plotted. The assays were repeated three times and the representatives are shown.

overexpressed (Fig. 1A). This effect of NEMO on the expression of $\gamma-\mathrm{GCS}_{\mathrm{H}}$ and $\gamma-\mathrm{GCS}_{\mathrm{L}}$ was also confirmed at the protein level by Western blot analysis (Fig. 1B).

To test whether the up-regulation of $\gamma$-GCS by NEMOmediated c-Myc stabilization is reflected by the change in the cellular GSH level, we next measured intracellular GSH level of the control and NEMO-overexpressing cells before and after IR treatment. The intracellular GSH level of control cells was decreased at $6 \mathrm{~h}$ after irradiation and then recovered to the basal level after $12 \mathrm{~h}$. In contrast, as expected from the elevated expression of $\gamma$-GCSs, the intracellular GSH level of NEMO-transfected cells was higher both before and after IR treatment with a negligible temporary decrease at $6 \mathrm{~h}$ (Fig. 2A). Irradiation of cells in general leads to the increase of intracel- lular ROS level. We reasoned that the elevated intracellular GSH level can modulate such a redox response to IR in NEMOtranfected cells. The increase of intracellular ROS level after IR treatment was significantly reduced in NEMO-transfected cells compared to the control cells, although the basal levels of intracellular ROS were almost identical between the control and NEMO-transfected cells (Fig. 2B). Taken together, c-Myc stabilization by NEMO overexpression causes up-regulation of $\gamma$-GCS expression resulting in the modulation of cellular redox response to IR, through the enhanced synthesis of GSH followed by reduced accumulation of intracellular ROS.

c-Myc stabilization by NEMO renders cells more resistant to $I R$. We then examined whether c-Myc stabilization by 

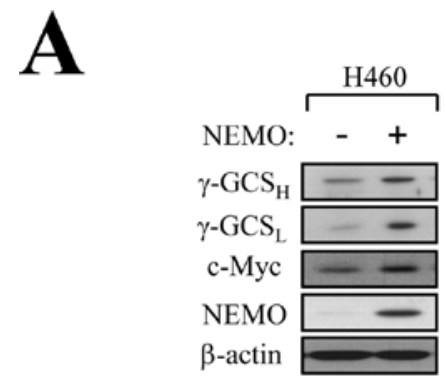

C
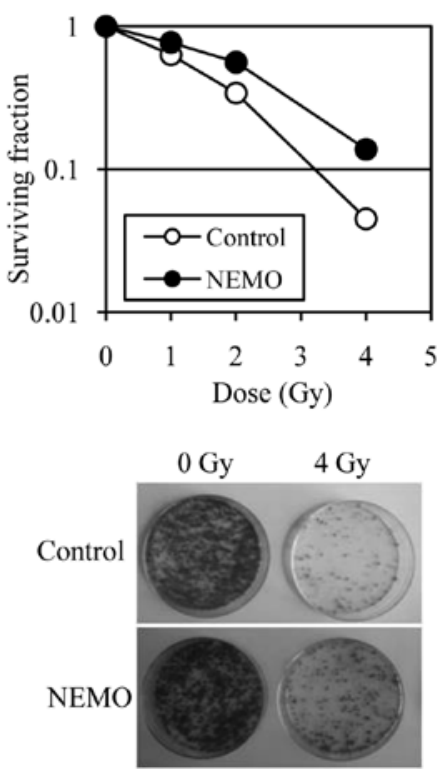

B

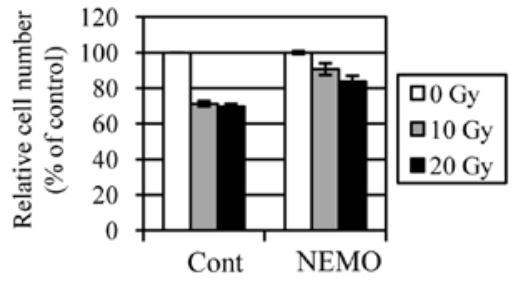

D
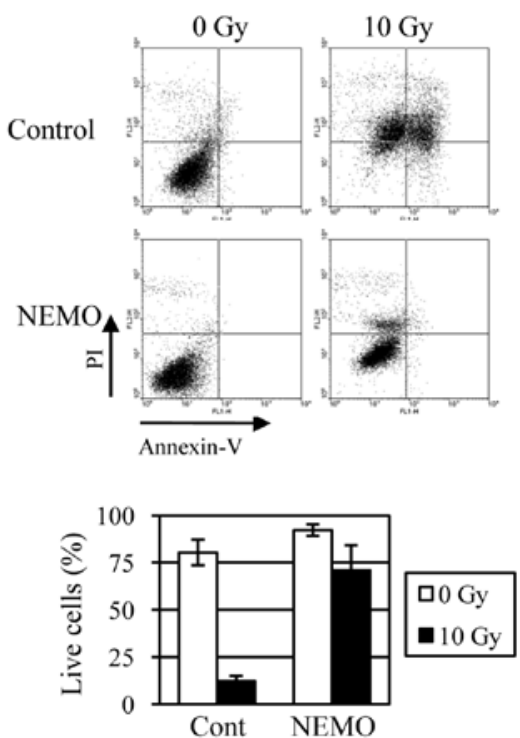

Figure 4. Stable overexpression of NEMO also exhibits the protective effects against IR in lung cancer H460 cells. (A) Confirmation of increased expression of $\gamma-\mathrm{GCS}_{\mathrm{H}}, \gamma-\mathrm{GCS}_{\mathrm{L}}$ and c-Myc by stable overexpression of NEMO in H460. The cell lysates prepared from the control or NEMO-overexpressing stable cell lines were analyzed by Western blotting. (B) NEMO alleviates IR-induced growth inhibition. The control or NEMO-overexpressing stable H460 cell lines were irradiated at the indicated doses and the relative viable cell numbers were determined $72 \mathrm{~h}$ after irradiation by MTS assay. (C) NEMO-overexpressing cells are less sensitive to IR in clonogenic survival assay. The control and NEMO-overexpressing stable H460 cell lines were seeded and irradiated at the indicated doses, then the surviving colonies were counted after 14 days. The surviving colony numbers were plotted in log scale against IR doses. The pictures for representative plates for 0 and 4 Gy treatments are also shown below. (D) Stable overexpression of NEMO protects H460 from IR-induced cell death. The control and NEMO-overexpressing stable H460 cell lines were irradiated at $10 \mathrm{~Gy}$ or not and cell death was determined after $48 \mathrm{~h}$ by Annexin V/PI staining.

NEMO affects cellular response to IR. For this purpose, we compared the growth inhibition and apoptosis induction by IR after transfection of a combination of NEMO and siRNA specific for c-Myc. c-Myc protein level was higher in NEMOtransfected cells as previously shown, and the elevated c-Myc level in NEMO-transfected cells could be almost completely removed by transfection of siRNA specific for c-Myc (Fig. 3A). To assess the effect of c-Myc stabilization by NEMO on growth inhibition, we carried out MTS cell proliferation assay. Growth of the control cells was inhibited by IR at a dose-dependent manner, while this growth-inhibitory effect of IR was almost completely blocked by NEMO transfection (Fig. 3B, left panel). This effect of NEMO transfection was largely abrogated when c-Myc siRNA had been additionally transfected (Fig. 3B, right panel). These results suggest that NEMO overexpression renders cells more resistant to IR through the up-regulation of c-Myc. To further support the effect of NEMO-mediated c-Myc stabilization on the cellular response to IR, we analyzed IR-induced apoptosis. Cells that had been transfected and irradiated as indicated were stained with Annexin V/PI and analyzed using FACS. Cells negative for both Annexin V and PI staining were assigned as undamaged live cells. In control cells, about $40 \%$ of cells were negative for both Annexin V and PI staining $48 \mathrm{~h}$ after irradiation, while about $77 \%$ of NEMO-transfected cells remained live after irradiation (Fig. 3C, left panel). Transfection of c-Myc siRNA significantly restored the apoptotic susceptibility to IR of NEMO-transfected cells (Fig. 3C, right panel), reconfirming the effect of NEMO-mediated c-Myc up-regulation. Altogether, these results suggest that the modification and stabilization of c-Myc by NEMO renders cells more resistant to IR.

To further demonstrate the effect of NEMO-mediated c-Myc stabilization on the radiosensitivity of cancer cells, we established stable cell line overexpressing NEMO from lung cancer H460 cells. Stable overexpression of NEMO in $\mathrm{H} 460$ induced c-Myc up-regulation and increased expression of $\gamma-\mathrm{GCS}_{\mathrm{H}}$ and $\gamma-\mathrm{GCS}_{\mathrm{L}}$ as expected (Fig. 4A). Growth 


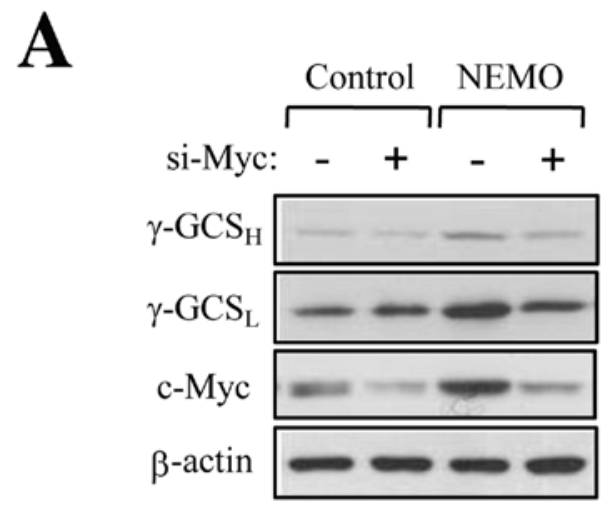

B

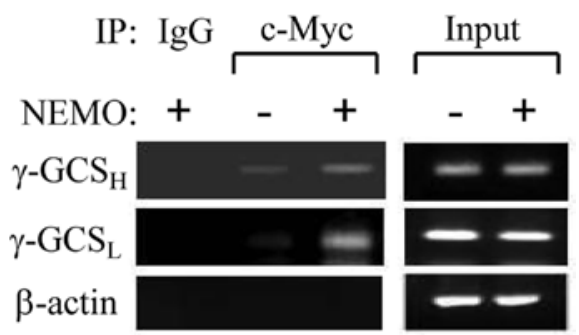

Figure 5. Up-regulation of $\gamma$-GCS by NEMO overexpression is dependent on c-Myc. (A) Knocking-down c-Myc by siRNA transfection blocks the increase of $\gamma$-GCS expression by NEMO overexpression. HEK293 cells were transfected with scrambled control or c-Myc specific siRNA in combination with the control or NEMO expression vector, and the expression level of each protein was analyzed by Western blotting. (B) NEMO enhances c-Myc recruitment to the promoters of $\gamma-\mathrm{GCS}_{\mathrm{H}}$ and $\gamma-\mathrm{GCS}_{\mathrm{L}}$. ChIP assay was carried out as described in Materials and methods.

inhibition by IR was significantly reduced when NEMO was overexpressed in H460 cells (Fig. 4B). We then performed colony-formation assay to examine the effect of c-Myc stabilization by NEMO on clonogenic cell survival in response to IR. The number of surviving colonies after irradiation was significantly higher in NEMO-overexpressing cell compared to the control cell (Fig. 4C), indicating that NEMO overexpression desensitized H460 cells to IR. Apoptosis analysis also showed that NEMO overexpression prevented apoptosis to a significant extent after irradiation in H460 (Fig. 4D).

Up-regulation of $\gamma$-GCS by NEMO overexpression is dependent on $c-M y c$. To confirm the role of $\mathrm{c}-\mathrm{Myc}$ in the up-regulation of $\gamma$-GCS by NEMO, we examined whether c-Myc down-regulation affects NEMO-induced $\gamma$-GCS increase. Augmented expression of $\gamma-\mathrm{GCS}_{\mathrm{H}}$ and $\gamma-\mathrm{GCS}_{\mathrm{L}}$ by NEMO overexpression returned to a basal level, when c-Myc expression was knocked-down by siRNA transfection (Fig. 5A). To examine whether the up-regulation of $\gamma$-GCS by NEMO overexpression is through facilitation of c-Myc binding to the promoters of $\gamma$-GCS, ChIP assay was carried out. The regions covering $\mathrm{E}$ boxes on the promoters of $\gamma-\mathrm{GCS}_{\mathrm{H}}$ and $\gamma-\mathrm{GCS}_{\mathrm{L}}$ were amplified by PCR from fragmented chromatins immunoprecipitated by specific c-Myc antibody. As expected, NEMO overexpression significantly enhanced the recruitment of c-Myc to the E boxes in the promoter regions of $\gamma-\mathrm{GCS}_{\mathrm{H}}$ and $\gamma-\mathrm{GCS}_{\mathrm{L}}$ (Fig. 5B).

\section{Discussion}

We have recently demonstrated that NEMO induces phosphorylation and stabilization of c-Myc through direct interaction in the nucleus (13). Since c-Myc plays critical roles in a variety of cellular processes such as cell cycle progression, proliferation, differentiation and apoptosis, the biological consequences of NEMO-mediated c-Myc phosphorylation/stabilization can be manifested in diverse ways according to the physiological context. As one of continued efforts to address the biological significance of this c-Myc phosphorylation/stabilization by NEMO, we planned to examine the effect on the cellular response to IR.

In this study, we demonstrated that NEMO overexpression activates $\gamma$-GCS expression transcriptionally by enhancing the recruitment of c-Myc to the regulatory regions on the promoters of $\gamma-\mathrm{GCS}_{\mathrm{H}}$ and $\gamma-\mathrm{GCS}_{\mathrm{L}}$. It has been elucidated that $\mathrm{c}-\mathrm{Myc}$ phosphorylation at Ser62 confers the promoter specificity of target genes, and is required for the activation of $\gamma$-GCS gene expression during the cellular response to oxidative stress (14). Our previous report showed that NEMO induces c-Myc phosphorylation at both Thr58 and Ser62 as well as protein stabilization (13). The present result showing $\gamma$-GCS up-regulation by NEMO is actually as expected considering these two previous reports. Ras-mediated mitogenic stimuli also converge into the c-Myc stabilization coupled with phosphorylation at Ser62 (4). Therefore, our results raise a question as to whether the interaction between NEMO and c-Myc, which causes phosphorylation and stabilization of c-Myc, is a part of the cascade events in the cellular response to oxidative stress or Ras-mediated mitogenic stimulation. This possibility needs to be investigated in the future.

As to cellular susceptibility to apoptotic stimuli, numerous reports have revealed two-faced functions of c-Myc. c-Myc induces apoptosis and sensitizes cells to various apoptotic stimuli $(12,15)$, but also has been reported to protect cells from apoptosis $(6,8,10)$. In this study, we report that c-Myc, when it is phosphorylated and stabilized by NEMO, protects cells from IR-induced cell death. At the same time, the expression of $\gamma$-GCS, a c-Myc target protein critical for defense from oxidative stress, was increased. This led to the increase of intracellular GSH level, and consequently alleviates IR-induced ROS increase. Phosphorylation and stabilization of c-Myc by NEMO was thus expected to exhibit protective effect against IR, since ROS generation in addition to DNA damage is one of the main mechanisms of IR-induced cell killing. In contrast, c-Myc is also known to increase ROS in some cases. c-Myc in cooperation with E2F1 potentiate apoptosis through inhibition of $\mathrm{NF}-\kappa \mathrm{B}$ activity that facilitates MnSOD-mediated ROS elimination (16). c-Myc activation can increase ROS, and induce DNA damage as a mechanism for oncogene-induced genetic instability (17). In these cases, $\gamma$-GCS may not be induced by c-Myc activation or the redoxcontrolling capacity of $\gamma$-GCS, if ever it is up-regulated by c-Myc activation, may be surpassed by ROS-increasing pressure under certain circumstances. Whether and how the NEMO-mediated c-Myc phosphorylation/stabilization 
influences cellular response to other stimuli remains to be answered.

Since the activity of c-Myc is an attractive target for cancer therapy, numerous efforts have been made to develop c-Myc inhibitors using various approaches, and several small molecules have been identified as an inhibitor of c-Mycinduced transformation $(18,19)$. Our data have demonstrated that interaction between c-Myc and NEMO comprises a novel mechanism to regulate $\mathrm{c}-\mathrm{Myc}$ function at least in the activation of $\gamma$-GCS expression making cells more resistant to IR, and thus suggest that the interaction between NEMO and c-Myc may serve as a good target for the development of strategy to overcome the resistance to radiotherapy.

\section{Acknowledgements}

This study was supported by a grant from Nuclear Research and Development Program funded by the Korean Ministry of Education, Science and Technology.

\section{References}

1. Hann SR and Eisenman RN: Proteins encoded by the human c-myc oncogene: Differential expression in neoplastic cells. Mol Cell Biol 4: 2486-2497, 1984

2. Salghetti SE, Kim SY and Tansey WP: Destruction of myc by ubiquitin-mediated proteolysis: cancer-associated and transforming mutations stabilize myc. EMBO J 18: 717-726, 1999.

3. Sears R, Leone G, DeGregori J and Nevins JR: Ras enhances myc protein stability. Mol Cell 3: 169-179, 1999.

4. Sears R, Nuckolls F, Haura E, Taya Y, Tamai K and Nevins JR: Multiple ras-dependent phosphorylation pathways regulate myc protein stability. Genes Dev 14: 2501-2514, 2000.

5. Nesbit CE, Tersak JM and Prochownik EV: MYC oncogenes and human neoplastic disease. Oncogene 18: 3004-3016, 1999.

6. Biroccio A, Benassi B, Amodei S, Gabellini C, Del Bufalo D and Zupi G: c-myc down-regulation increases susceptibility to cisplatin through reactive oxygen species-mediated apoptosis in M14 human melanoma cells. Mol Pharmacol 60: 174-182, 2001.
7. Biroccio A, Benassi B, Filomeni G, et al: Glutathione influences c-myc-induced apoptosis in M14 human melanoma cells. J Biol Chem 277: 43763-43770, 2002.

8. Gatti G, Maresca G, Natoli M, Florenzano F, Nicolin A, Felsani A and D'Agnano I: MYC prevents apoptosis and enhances endoreduplication induced by paclitaxel. PLoS One 4: e5442, 2009.

9. Skvortsova I, Popper BA, Skvortsov S, et al: Pretreatment with rituximab enhances radiosensitivity of non-Hodgkin's lymphoma cells. J Radiat Res 46: 241-248, 2005.

10. Bucci B, D'Agnano I, Amendola D, et al: Myc down-regulation sensitizes melanoma cells to radiotherapy by inhibiting MLH1 and MSH2 mismatch repair proteins. Clin Cancer Res 11: 2756-2767, 2005

11. Maclean KH, Keller UB, Rodriguez-Galindo C, Nilsson JA and Cleveland JL: c-myc augments gamma irradiation-induced apoptosis by suppressing bcl-XL. Mol Cell Biol 23: 7256-7270, 2003.

12. Juin P, Hunt A, Littlewood T, Griffiths B, Swigart LB, Korsmeyer S and Evan G: c-myc functionally cooperates with bax to induce apoptosis. Mol Cell Biol 22: 6158-6169, 2002.

13. Kim BY, Yang JS, Kwak SY, Zhang XK and Han YH: NEMO stabilizes c-myc through direct interaction in the nucleus. FEBS Lett 584: 4524-4530, 2010.

14. Benassi B, Fanciulli M, Fiorentino F, et al: c-myc phosphorylation is required for cellular response to oxidative stress. Mol Cell 21: 509-519, 2006

15. Evan GI, Wyllie AH, Gilbert CS, et al: Induction of apoptosis in fibroblasts by c-myc protein. Cell 69: 119-128, 1992.

16. Tanaka H, Matsumura I, Ezoe S, et al: E2F1 and c-myc potentiate apoptosis through inhibition of NF-kappaB activity that facilitates MnSOD-mediated ROS elimination. Mol Cell 9: 1017-1029, 2002.

17. Vafa O, Wade M, Kern S, Beeche M, Pandita TK, Hampton GM and Wahl GM: c-myc can induce DNA damage, increase reactive oxygen species, and mitigate p53 function: a mechanism for oncogene-induced genetic instability. Mol Cell 9: 1031-1044, 2002.

18. Mo $\mathrm{H}$ and Henriksson $\mathrm{M}$ : Identification of small molecules that induce apoptosis in a myc-dependent manner and inhibit mycdriven transformation. Proc Natl Acad Sci USA 103: 6344-6349, 2006.

19. Berg T, Cohen SB, Desharnais J, et al: Small-molecule antagonists of Myc/Max dimerization inhibit myc-induced transformation of chicken embryo fibroblasts. Proc Natl Acad Sci USA 99: 3830-3835, 2002. 